\title{
Review of sleep studies of patients with chronic insomnia at a sleep disorder unit
}

\author{
Sheau-Hwa Wong $^{1}$, MBBS, MMed, Beng-Yeong Ng$^{1}$, MBBS, MMed
}

\begin{abstract}
INTRODUCTION Chronic insomnia is associated with many physical and psychiatric illnesses, and its underlying aetiology needs to be identified in order to achieve safe and effective treatment. Obstructive sleep apnoea (OSA) and periodic limb movement disorder (PLMD) are common primary sleep disorders that can lead to chronic insomnia. Patients with these conditions are evaluated using polysomnography (PSG).

METHODS The PSG records of 106 patients with chronic insomnia who presented to a multidisciplinary sleep clinic in Singapore over a five-year period were reviewed. To examine the utility of PSG in the evaluation of chronic insomnia, the clinical diagnoses of the patients before and after the sleep studies were compared.

RESULTS Among the 106 patients, 69 (65.4\%) were suspected to have primary sleep disorders based on clinical history and examination alone. Following PSG evaluation, $42.5 \%$ and $4.7 \%$ of the study population were diagnosed with OSA and PLMD, respectively. OSA was found in $35.9 \%$ of the 39 patients who had underlying psychiatric conditions.

CONCLUSION This study illustrates that many patients with chronic insomnia have underlying primary sleep disorders. It also highlights the danger of attributing chronic insomnia in psychiatric patients to their illness, without giving due consideration to other possible aetiologies. Clinicians should maintain a high index of suspicion for the presence of other aetiologies, and make timely and targeted referrals for sleep studies where appropriate.
\end{abstract}

Keywords: chronic insomnia, obstructive sleep apnoea, polysomnography, Singapore

\section{INTRODUCTION}

Insomnia is the most commonly reported sleep problem in industrialised countries worldwide. ${ }^{(1-4)}$ An international survey on sleep problems in the general population showed a high prevalence of insomnia (e.g. $23 \%$ in Japan, $56 \%$ in the United States) and that many individuals with insomnia $(47 \%-67 \%)$ did not seek medical attention for their sleep difficulties. ${ }^{(5)}$ Among those who sought medical help, 50\%-90\% received treatment. Thus, insomnia remains a largely underdiagnosed and under-treated problem.

The situation in Singapore is similar, as shown in a 2002 general population survey on insomnia, ${ }^{(6)}$ in which 200 out of 430 randomly selected persons (aged 18-64 years) were found to have difficulty sleeping. Only $10 \%$ of these 200 persons sought medical advice and almost $50 \%$ of them underplayed the significance of their sleep difficulties. Among the 200 persons, $65 \%$ believed that sleeping pills were unsafe to use and $82 \%$ were worried about becoming addicted to them. ${ }^{(6)}$ In comparison, a study conducted by Yeo et al in 1996 found that the prevalence of insomnia in Singapore was $15.3 \%$ (male $12.9 \%$, female $17.5 \%$ ). ${ }^{(7)}$ In that study, $86 \%$ of the individuals who complained about sleep problems were not on treatment and only $21.6 \%$ had sought medical advice.

Descriptions of the term 'insomnia' are often subjective, open to interpretation and coloured by individual expectations. While the condition can be objectively studied through polysomnography (PSG) to monitor various physiological markers in a sleeping individual, this is not a cost-effective or practical method in day-to-day clinical practice. Assessment based solely on a patient's verbal report is also inadequate, as sleep quality is subjective and can vary considerably among individuals.

While transient and short-term insomnia is an almost universal complaint, persistent insomnia in a seemingly healthy individual may be a presenting symptom of a more serious illness that is of a medical and/or psychiatric nature. Identifying the underlying reason for persistent insomnia plays an important role in the treatment of the condition. Persistent insomnia is often attributed to psychological causes such as mental illness. There is some basis for this attribution, as about $40 \%$ of all insomnia cases are associated with common psychiatric conditions such as depression or anxiety. ${ }^{(8-10)}$ Unfortunately, the stigma associated with mental illness remains a significant barrier to help-seeking. In the second edition of the International Classification of Sleep Disorders (ICSD-2), there are 55 disorders that include insomnia as one of the complaints. ${ }^{(11)}$ The ICSD-2 diagnostic framework also identifies categories of sleep disorders that are secondary to medical conditions, substance use and psychiatric disorders. Therefore, while underlying psychiatric disorders are often alleged to be the culprit in chronic insomnia, the attending physician should be vigilant to other contributing factors such as the patient's underlying medical problems, medications used for treatment and possible substance abuse/dependence.

Obstructive sleep apnoea (OSA) is the most common primary sleep disorder, with an estimated prevalence of $2 \%-5 \%$ in women and $3 \%-7 \%$ in men. ${ }^{(12)}$ OSA should be considered in patients with chronic insomnia since a high prevalence (39\%-58\%) of insomnia

${ }^{1}$ Department of Psychiatry, Singapore General Hospital, Singapore

Correspondence: DrSheau-HwaWong, Visiting Consultant, Department of Psychiatry, Singapore General Hospital, 10 Outram Road, Singapore169037.wong.sheau.hwa@sgh.com.sg 
symptoms has been observed in patients with OSA in previous studies. ${ }^{(13)}$ Furthermore, $29 \%-67 \%$ of patients with insomnia have an apnoea-hypopnoea index greater than 5, indicating the presence of at least a mild degree of sleep-disordered breathing (SDB). ${ }^{(13)}$ In a 2001 study by Krakow et al, around $50 \%$ of the patients with a diagnosis of SDB reported insomnia symptoms, ${ }^{(14)}$ including difficulty in initiating sleep, difficulty in staying asleep, longer mean sleep onset latency and impaired sleep efficiency. In OSA patients, Chung suggested that sleep initiation difficulties may be due to a state of hyperarousal, while sleep maintenance difficulties and early awakenings may be due to sleep fragmentation and frequent arousals from the effects of SDB. ${ }^{(15)}$ A recent study showed that Asians may have a higher predisposition to OSA than Caucasians due to craniofacial profile differences. ${ }^{(16)}$ Although Asian populations have a lower incidence of obesity, they have similar prevalence rates for OSA as Caucasian populations. In other words, the absence of obesity in an Asian patient should not mislead physicians into dismissing OSA as a diagnosis.

Overnight PSG is a standard tool in sleep medicine for evaluating sleep-related pathophysiology. However, its role in evaluating insomnia has been controversial. ${ }^{(17)}$ The American Academy of Sleep Medicine's practice parameters for using PSG recommend against the use of routine PSG in clinical evaluation of transient or chronic insomnia. ${ }^{(18)}$ It states that insomnia is primarily diagnosed by clinical evaluation, through detailed medical, psychiatric and sleep history-taking. However, it outlined situations where PSG assessment may be appropriate, including those in which: (a) a diagnosis is uncertain; (b) sleep-related breathing or periodic limb movement disorder (PLMD) is suspected; (c) the patient is refractory to insomnia treatment; (d) violent behaviours are comorbid with insomnia; or (e) there are circadian rhythm disruptions that complicate the clinical picture. It also states that routine PSG is not useful for evaluating insomnia that is attributable to psychiatric conditions, dementia, fibromyalgia syndrome and chronic fatigue syndrome.

Insomnia patients are usually managed by primary care physicians and not sleep specialists. In a local study conducted by Mahendran et al in 2007, the authors reviewed all patients who were referred to the insomnia clinic of a tertiary psychiatric hospital over a three-year period. The study found that $52.9 \%$ of the patients who were referred to the insomnia clinic attended due to the insistence of the referring physician and that $54.2 \%$ of them had had sleep problems for more than a year. Additionally, $74.1 \%$ of the patients had received prior treatment for their sleep complaints and $90.5 \%$ had been prescribed hypnotics; $52.5 \%$ were diagnosed with a primary psychiatric disorder, the most common being generalised anxiety disorder (14.2\%), followed by obsessive compulsive disorder (9.9\%) and depression/ dysthymia $(9.9 \%)$. The remainder $(47.5 \%)$ were diagnosed with primary insomnia. Other conditions included alcohol abuse (15.6\%) and substance abuse, including prescription drug abuse (10.0). ${ }^{(19)}$ However, as the insomnia clinic in that study was based in a tertiary psychiatric hospital, it is unclear if insomnia patients referred to other specialty clinics (such as otolaryngology) may have different characteristics.

With this background in mind, we reviewed PSGs that were conducted in patients with chronic insomnia, to examine the diagnostic outcomes and the utility of PSG in their evaluation.

\section{METHODS}

We reviewed sleep studies conducted on 106 chronic insomnia patients at the multidisciplinary sleep disorder unit (SDU) of the Singapore General Hospital from 2006 to 2010. Patients' PSG indices and diagnoses were tabulated and cross-referenced to medical records to identify the reasons the sleep studies were conducted. Pre- and post-PSG diagnoses were compared to examine the utility of targeted PSG in the evaluation of chronic insomnia. Information regarding comorbid psychiatric conditions was also collected to examine how these conditions influenced the management of insomnia. Patients who were referred for evaluation of their insomnia due to suspected primary sleep disorders or other causes were not included in this review. This allowed us to examine whether the referring doctors had missed underlying sleep disorders in chronic insomnia patients during clinical history-taking and physical examination.

The sleep studies were conducted using 12-channel attended PSG suites, and scores were given according to the criteria set by Rechtschaffen and Kales. ${ }^{(20)}$ Patients undergoing PSG were asked to avoid taking any psychoactive medications or hypnotics, which may alter sleep physiology and lead to artificial findings. If the patients were not able to sleep, zolpidem was prescribed, as it is least likely to alter sleep architecture and muscular tone. The unit, which is staffed by psychiatrists, neurologists, respiratory physicians, otolaryngologists, clinical psychologists, polysomnographic technologists and respiratory therapists, receives referrals from primary care physicians in the community and other medical professionals (e.g. psychiatrists from psychiatric hospitals that are not equipped with PSG facilities); it also admits self-referred patients. As the patients involved in the present study had undergone evaluations and treatment as per usual practice, the results of this study can represent the management of insomnia patients in a real-world situation. The study was approved by the hospital's institutional review board, and consent requirements were waived, as it did not require direct patient interaction.

\section{RESULTS}

All 106 patients in the sleep studies reviewed were found to have suffered from insomnia for at least six months. Their mean age was 44.8 (range 19-81) years. The distribution of patients was fairly even between the ages of 20 and 69 years, and there were slightly more male patients (58.5\%; Table I). Among the 106 patients, 39 (36.8\%) were found to have underlying psychiatric conditions. In terms of ethnic composition, $84.0 \%$ of the patients were of Chinese descent. When compared to the general population in Singapore (Malay 13.9\%, Indian 7.9\%), (21) there was a slight overrepresentation of patients of Indian 
Table I. Characteristics of the study population $(n=106)$.

\begin{tabular}{|c|c|}
\hline Variable & No. (\%) \\
\hline \multicolumn{2}{|l|}{ Age (yr) } \\
\hline$<20$ & $7(6.6)$ \\
\hline $20-29$ & $14(13.2)$ \\
\hline $30-39$ & $20(18.9)$ \\
\hline $40-49$ & $22(20.8)$ \\
\hline $50-59$ & $20(18.9)$ \\
\hline $60-69$ & $18(17.0)$ \\
\hline $70-79$ & $4(3.8)$ \\
\hline $80-89$ & $1(0.9)$ \\
\hline \multicolumn{2}{|l|}{ Gender } \\
\hline Male & $62(58.5)$ \\
\hline Female & $44(41.5)$ \\
\hline \multicolumn{2}{|l|}{ Ethnicity } \\
\hline Chinese & $89(84.0)$ \\
\hline Malay & $3(2.8)$ \\
\hline Indian & $11(10.4)$ \\
\hline Others & $3(2.8)$ \\
\hline \multicolumn{2}{|l|}{ Nationality } \\
\hline Singaporean/permanent resident & $102(96.2)$ \\
\hline Foreigner & $4(3.8)$ \\
\hline \multicolumn{2}{|l|}{ BMI $\left(\mathrm{kg} / \mathrm{m}^{2}\right)^{*}$} \\
\hline$<18.5$ & $9(8.5)$ \\
\hline $18.5-22.9$ & $37(34.9)$ \\
\hline $23.0-27.4$ & 35 (33.0) \\
\hline$>27.4$ & $24(22.6)$ \\
\hline
\end{tabular}

*The data of one patient was missing. BMI: body mass index

ethnicity $(10.4 \%)$ and an underrepresentation of patients who were of Malay ethnicity (2.8\%).

Psychiatrists attended to about half $(\mathrm{n}=58,54.7 \%$ ) of the referred patients, while various other specialists (predominantly neurologists) attended to the remaining referred patients $(n=48$, 45.3\%). Between 2006 and 2010, there was an increase in the number of psychiatrists who supported services at the SDU, in tandem with the rise in number of cases seen by psychiatrists over the same period. There was also a gradual increase in the number of new cases seen by other specialists, although their staff numbers remained the same (Table II).

Prior to PSG, at least 69 (65.4\%) of the patients had enough clinical features in their history and physical examination for the attending specialists to suspect the presence of primary sleep disorders. These features included heavy snoring or choking during sleep, unrefreshing sleep with severe daytime fatigue, awakenings due to limb jerks, elevated body mass index $\left(>30 \mathrm{~kg} / \mathrm{m}^{2}\right)$ and large neck circumference $(>43 \mathrm{~cm})$. The referring doctors either missed these features or did not indicate them in their referral memos. OSA was suspected in 53 (50.0\%) patients and PLMD was suspected in $16(15.1 \%)$ patients. Although primary insomnia was provisionally diagnosed in $23(21.7 \%)$ patients, sleep studies were conducted to rule out possible primary sleep disorders in these patients. The remaining 14 patients had various other suspected conditions, such as narcolepsy and rapid eye movement sleep behaviour disorder.
Table II. Case load (all causes) and staff numbers at Singapore General Hospital's sleep disorder unit from 2006 to 2010.

\begin{tabular}{cccccccc}
\hline Year & \multicolumn{3}{c}{ Psychiatrists } & & \multicolumn{3}{c}{ Other specialists* } \\
\cline { 2 - 4 } \cline { 7 - 8 } & $\begin{array}{c}\text { Staff } \\
\text { strength }\end{array}$ & $\begin{array}{c}\text { New } \\
\text { cases } \\
\text { seen }\end{array}$ & $\begin{array}{c}\text { Study } \\
\text { patients } \\
\text { seen }\end{array}$ & & $\begin{array}{c}\text { Staff } \\
\text { strength }\end{array}$ & $\begin{array}{c}\text { New } \\
\text { cases } \\
\text { seen }\end{array}$ & $\begin{array}{c}\text { Study } \\
\text { patients } \\
\text { seen }\end{array}$ \\
\hline 2006 & 1 & 78 & 11 & & 9 & 537 & 12 \\
2007 & 1 & 51 & 5 & & 9 & 522 & 14 \\
2008 & 1 & 62 & 10 & & 9 & 579 & 9 \\
2009 & 3 & 124 & 7 & & 9 & 639 & 5 \\
2010 & 5 & 289 & 25 & & 9 & 613 & 8 \\
\hline
\end{tabular}

Data presented as number. *Includes neurologists, respiratory physicians and otolaryngologists.

Table III. Number of patients found to have obstructive sleep apnoea (OSA) in the study population $(n=106)$.

\begin{tabular}{lcc}
\hline $\begin{array}{l}\text { Apnoea-hypopnoea } \\
\text { index }\end{array}$ & \multicolumn{2}{c}{ No. (\%) } \\
\cline { 2 - 3 } & $\begin{array}{c}\text { Suspected* } \\
\text { and OSA found } \\
\text { (true positive) }\end{array}$ & $\begin{array}{c}\text { Not suspected } \\
\text { but OSA found } \\
\text { ('missed' cases) }\end{array}$ \\
\hline $5-15$ (mild) & $4(3.8)$ & $10(9.4)$ \\
$16-30$ (moderate) & $11(10.4)$ & $2(1.9)$ \\
$>30$ (severe) & $15(14.2)$ & $3(2.8)$ \\
Total & $30(28.3)$ & $15(14.2)$ \\
\hline
\end{tabular}

*53 patients were suspected to have OSA based on clinical history and physical examination alone.

The mean sleep efficiency was $73.9 \% \pm 19.2 \%$, indicating overall poor sleep in our study population. Most $(n=67,63.2 \%)$ of the patients were found to have poor sleep efficiency. Among these 67 patients, 15 did not have a diagnosable primary sleep disorder based on PSG that could account for poor sleep efficiency, which suggested a diagnosis of primary insomnia, and 13 patients similarly had no diagnosable primary sleep disorder based on PSG, but had underlying psychiatric conditions that may have contributed to poor sleep efficiency. Among the 106 patients, 29 (27.4\%) patients had a sleep onset latency that was longer than 30 minutes; ten of these 29 patients had underlying psychiatric conditions. In our study population, the rate of prolonged sleep onset latency in psychiatric patients was found to be similar to that in non-psychiatric patients.

A total of $45(42.5 \%)$ patients in our study population showed evidence of OSA upon PSG; 30 of them were among the 53 patients who were suspected to be suffering from OSA based on clinical history and physical examination, and 15 were 'missed' cases who were not originally suspected to have OSA (Table III). Notably, five of these 15 'missed' patients had moderately severe to severe OSA. Therefore, medical history and physical examination alone were not sufficient to detect OSA, even when it is severe.

PLMD can cause frequent arousals, leading to sleep fragmentation. Of the 16 patients who were clinically suspected to have PLMD, only 5 (31.3\%) had positive PSG findings for PLMD. In total, $4.7 \%$ of the study population were found to have PLMD. However, 23 patients who were not originally suspected to have PLMD were found to have an elevated periodic limb movement 
index (PLMI) of more than five per hour. The clinical significance of this is uncertain and could represent incidental findings.

Among the 106 patients, 39 (36.8\%) were found to have underlying psychiatric conditions (mostly anxiety disorder, depression or mixed anxiety and depression). Non-psychiatrists attended to approximately half of this group of patients for their insomnia complaints. Table IV provides an overview of the diagnostic outcomes of this group of patients after PSG. Various degrees of OSA were found in 14 (35.9\%) patients in this group, of whom nine had moderate to severe OSA. Isolated raised PLMI with poor sleep efficiency was found in 6 (15.4\%) patients; 7 (17.9\%) patients showed no PSG evidence of OSA or raised PLMI and had normal sleep efficiency, suggesting possible sleep state misperception.

Of the 23 patients originally suspected to have primary insomnia, 8 (34.8\%) showed poor sleep efficiency but no PSG evidence of primary sleep disorders, and 7 (30.4\%) patients were found to have normal sleep efficiency with no PSG evidence of primary sleep disorders, suggesting possible sleep state misperception or conditioned insomnia.

\section{DISCUSSION}

The number of patients referred to the SDU of Singapore General Hospital for insomnia has been increasing steadily. This could be due to the introduction of Clinical Practice Guidelines (CPGs) for the prescription of benzodiazepines ${ }^{(22)}$ by the Ministry of Health in 2008, which resulted in general practitioners making more patient referrals rather than prescribing potentially addictive hypnotics.

With the increase in the number of psychiatrists supporting the SDU, patients referred for insomnia have been preferentially channelled to their clinics; since 2010, almost all patients referred to the SDU for only the stated complaint of insomnia were sent to psychiatrists. Psychiatrists have a theoretical advantage over other specialists in assessing patients with insomnia, as they have an additional repertoire of clinical skills to assess psychological issues that may have contributed to the condition of these patients. Unfortunately, of the 289 new insomnia patients seen by psychiatrists in 2010 (Table II), only 25 underwent PSG for further evaluation. Anecdotally, many more patients would have benefitted from PSG evaluation but declined it, citing cost and inconvenience as reasons. Symptoms and features suspicious of primary sleep disorders can often be elicited in these patients. The referring primary care physician may not have identified these symptoms, or did not indicate them in the referrals. There were also patients who had no clear psychological reasons for their sleep complaints and were not responding to sleep medications. In such cases, a recommendation for a sleep study is warranted.

In the present study, almost $75 \%$ of the patients were 30 to 69 years old. Patients in this age group are more likely to be economically active and stable, as compared to those in their twenties (who are likely at the start of their careers). They may be more concerned about the impact of insomnia on their health and less tolerant to the physical effects of chronic insomnia on daytime function (e.g. reduced job performance). This explanation could only be based on reasonable assumptions, as the present
Table IV. Overview of the diagnostic outcomes after polysomnography (PSG) in patients with underlying psychiatric conditions $(n=39)$.

\begin{tabular}{lccc}
\hline Diagnostic outcome & \multicolumn{3}{c}{ No. (\%) } \\
\cline { 2 - 4 } & $\begin{array}{c}\text { Attended } \\
\text { to by a } \\
\text { psychiatrist } \\
\mathbf{( n = 2 0 )}\end{array}$ & $\begin{array}{c}\text { Attended } \\
\text { to by other } \\
\text { specialists* } \\
\mathbf{( n = 1 9 )}\end{array}$ & $\begin{array}{c}\text { Total } \\
(\mathbf{n}=\mathbf{3 9 )}\end{array}$ \\
\hline $\begin{array}{l}\text { Insomnia attributed to } \\
\text { psychiatric condition }\end{array}$ & $5(25.0)$ & $7(36.8)$ & $12(30.8)$ \\
$\begin{array}{l}\text { OSA } \\
\text { Raised PLMI }\end{array}$ & $7(35.0)$ & $7(36.8)$ & $14(35.9)$ \\
$\begin{array}{l}\text { Sleep state } \\
\text { misperception }\end{array}$ & $3(15.0)$ & $3(15.8)$ & $6(15.4)$ \\
\hline
\end{tabular}

*Includes neurologists, respiratory physicians and otolaryngologists. †Although there was no PSG evidence of obstructive sleep apnoea (OSA) or raised periodic limb movement index (PLMI), sleep efficiency was less than $85 \%$, suggesting that insomnia was possibly linked to the psychiatric condition. ${ }_{\text {NNo }}$ PSG evidence of OSA or raised PLMI and normal sleep efficiency (i.e. > 85\%).

study is a retrospective one and information about the patients' employment and socioeconomic status (obtained from their case records) was scant. Similarly, the observation that slightly more male patients underwent the sleep study as compared to female patients could be related to employment status. It is possible that some women homemakers did not view daytime impairment from insomnia to be as critical as those who would be appraised negatively for poor work performance. As such, these women might not have seen the need for a sleep study, but instead made behavioural changes (e.g. taking more naps in the day) to adapt. While current evidence shows that chronic insomnia is more common in elderly and female populations, ${ }^{(23)}$ this is in reference to the general population and not a clinical population, as in the present study.

The underrepresentation of patients of Malay ethnicity in the present study $(2.8 \% \text { vs. general population } 13.9 \%)^{(19)}$ was also found in a local study by Mahendran. ${ }^{(24)}$ In that study, patients of Malay ethnicity formed only $5.9 \%$ of all patients seen in the insomnia clinic of a psychiatric hospital. However, Yeo et al, in their study on insomnia in the community, found that patients of Malay ethnicity were more likely to have complaints of insomnia $(28.4 \%$ of patients with insomnia were Malay)..$^{(7)}$ Collectively, these findings, rather than showing a lower prevalence of insomnia in the Malay community, suggest that the Malay population adopts a different attitude toward seeking help for sleep problems.

An overview of the PSG sleep indices revealed that our study population had poor sleep characteristics in general. The mean scores for the different sleep indices (measuring sleep quantity, sleep efficiency, arousals, respiratory abnormalities and motor activities) were in the impaired or abnormal range for all 106 patients. In the present study, only 23 (21.7\%) patients were suspected of having primary insomnia based on clinical consultation. In contrast, this number was $47.5 \%$ in the study conducted by Mahendran et al. ${ }^{(19)}$ The proportion of patients in SDU who have primary insomnia is likely to be even higher, as many patients who were clinically diagnosed with primary 
insomnia may have declined PSG studies. About one-third of the 23 patients who were originally suspected to have primary insomnia had incidental findings on PSG that suggested the presence of a sleep disorder.

Of significance in the present study was the discovery of OSA in 45 patients, of which 15 were incidental findings through PSG evaluation (Table III). Of these 15 cases, ten had OSA of mild severity, which could explain why there was no suspicion of OSA: the patients may not have shown the typical symptoms or partner-reported observations that are seen in more severe cases. Among the five patients with moderate to severe OSA, two had body mass indices that were within the healthy range and neck circumferences that were not significantly thick. The presence of a high body mass index and a thick neck circumference would have raised suspicion of OSA.

It was interesting to note that, in the present study, the Epworth Sleepiness Scale (ESS) was administered to only 22 of the 106 patients with insomnia. ESS is a tool that has been validated for the assessment of OSA. ${ }^{(25)}$ While it can be argued that patients with insomnia would be expected to have high ESS scores due to their lack of rest (and hence excessive daytime sleepiness), the administration of ESS would nonetheless provide information on the severity of the extent of daytime dysfunction of insomnia sufferers. Repeated assessment could also help in assessing treatment effectiveness. In a recent study by Glidewell et al in which the Insomnia Severity Index was used in combination with ESS to identify patients with OSA, the authors reported that insomnia may, on its own, serve as an independent predictor for OSA in the primary care setting. ${ }^{(26)}$ Bjornsdottir et al also reported that OSA patients who suffer from insomnia scored less well on ESS and that women with OSA and insomnia were more likely to have sleep initiation difficulties. ${ }^{(27)}$ The findings of the present study highlight that, while insomnia is a nonspecific symptom, OSA remains one of the conditions that clinicians need to consider, particularly if there are relevant features in the patient history and ESS scores are high.

In our study population, five patients were diagnosed with PLMD after PSG. Raised PLMI (more than five per hour) was found in 23 other patients; however, this finding was deemed to be clinically insignificant in most of these 23 cases. Clinical interpretation had to be performed with care in this group of chronic insomnia patients, as many may have received prior drug treatment for their sleep symptoms. Selective serotonin reuptake inhibitors and tricyclic antidepressants, which are sometimes used for their sedating effects, are known to induce leg movements in sleep, leading to raised periodic leg movement readings. The number of diagnosed PLMD cases in our study population was unexpectedly low, given that the prevalence of PLMD in the general population is estimated to be around $4 \%$, with its incidence increasing with age. ${ }^{(28)}$ More PLMD cases were also expected, since insomnia is a common presentation for PLMD. However, if the ICSD-2 minimal criteria (PLMI of more than five per hour and insomnia associated with daytime dysfunction) are applied in the present study, 23 more patients would qualify for the diagnosis of PLMD.
Close to $40 \%$ of our study population were found to have underlying psychiatric disorders, consistent with the findings of other studies that examined patients with insomnia. In our subgroup of patients with underlying psychiatric disorders $(n=39)$, the likelihood of psychiatrists or other specialists attending to them was almost equal. This is not unexpected as such background information may not have been available to the triage team when the SDU received the referral. We found that 14 (35.9\%) of the 39 patients had OSA, with nine having moderate to severe OSA. Clinicians should, therefore, be conscientious when evaluating patients with underlying psychiatric disorders; the presence of a psychiatric illness should not distract them from a holistic evaluation of insomnia as a presenting problem. Current research provides evidence for a comorbid and bidirectional relationship between OSA and depression. ${ }^{(29)}$ Patients with untreated OSA are found to have higher rates of depressive syndromes, and OSA may be responsible for poor response to depression treatment. Conversely, undiagnosed OSA may be exacerbated by adjunct pharmaceutical drugs that are used to treat depression and insomnia. Benzodiazepines, which are commonly used for treating depression and insomnia, can worsen OSA; they can exacerbate airway obstruction (via the lowering of muscle tone), cause respiratory suppression and result in a higher hypercapnic threshold for arousal-ventilatory response during apnoeic episodes. In a review paper, up to $20 \%$ of patients who presented with depressive syndromes had OSA. Similarly, up to $20 \%$ of OSA patients were found to have depression. ${ }^{(29)}$ Serotonin was identified as the common neurobiological agent in both OSA and depression, with the two conditions having many shared risk factors such as metabolic syndrome, cardiovascular disease, hypertension and diabetes mellitus.

The present study was not without limitations. The population in this retrospective study was highly selective and may represent patients who have severe distress and/or dysfunction from their sleep disturbances, or have poor tolerance to such distress. Referral bias is significant in the present study, as the patients identified were highly self-selected and agreed to PSG evaluation. Some patients declined further evaluation with PSG due to economic reasons or contentment with 'quick-fix' medications, even though their clinical histories were suspicious for the presence of sleep disorders.

Most of the clinical data in the present study was obtained retrospectively from patient medical records. Data obtained via this method is neither comprehensive nor intentional, as compared to data obtained in a prospective study. Future studies reviewing the sleep studies of patients at the SDU should be prospective in design and include a control group. Chronic insomnia patients who did not undergo PSG could be used as controls, which was not done in the present study. In addition, standardised symptom rating scales and screening questionnaires could have been used to better characterise the patients.

In the present study, information on psychological symptoms and assessments of their severity and impact on insomnia symptoms were heterogeneous and lacked standardisation. Unlike physiological and categorical parameters, which are 
clear-cut, descriptions of psychological and mental states leave room for interpretation, resulting in poorer reliability and the possibility of confounding the clinical picture. Psychometric measures, such as the Beck Depression Inventory score and the Beck Anxiety Inventory score, would have been useful for providing some insight on how psychological factors contributed to patients' sleep disturbances. These measures would be useful in clarifying the nature of insomnia (between comorbid insomnia, in which sleep disturbances may persist, and insomnia secondary to psychiatric illness, which may improve with stabilisation of the patient's mental state). Records for comorbid medical conditions and medications used were of heterogeneous integrity, further confounding the clinical picture. The strategy with which psychiatrists approach patients who present with insomnia may also have differed from that of non-psychiatrist sleep specialists (due to different emphases and concerns). This may have influenced clinical management and stage of illness at which patients entered PSG evaluation. However, in spite of these limitations, the present study provides important lessons that are applicable in clinical practice because it was based on the observations of a real-world practice.

To conclude, the management of insomnia remains an interesting challenge for physicians. Insomnia is often treated symptomatically at the primary care level even though it is a complex medical condition that may have various underlying aetiologies, resulting in its chronicity. A high proportion of chronic insomnia sufferers have underlying psychiatric illnesses that pose as red herrings, leading to delayed or underdiagnosis of primary sleep disorders, which require different treatments from psychiatric illnesses.

In the present study, targeted use of PSG revealed the presence of common sleep disorders, especially OSA, in a significant proportion (up to one third) of chronic insomnia sufferers. Similar findings were also noted of the insomnia patients with underlying psychiatric conditions. We also found that, while at least $50 \%$ of the referred patients had sufficient symptoms and features to suggest the presence of primary sleep disorders, they were not identified by the referring physicians. This finding is a cause for concern, as it suggests that the referring physicians had inadequate knowledge and training in sleep medicine to effectively manage chronic insomnia. Physicians should have a comprehensive understanding of the aetiologies of insomnia and be able to make timely referrals for further investigations or goal-directed specialist interventions. This gap in sleep medicine education should be addressed by the medical education system. ${ }^{(30)}$

To mitigate this, physicians should consider using available CPGs to facilitate the diagnosis and management of sleep disorders. An example is the Toward Optimized Practice (TOP) CPG for management of adult insomnia by the Alberta Medical Association. TOP includes the Insomnia Screening Questionnaire, a 17-item tool that outlines six diagnostic domains, including SDB and other primary sleep disorders. ${ }^{(31)}$ The TOP programme provides a more comprehensive approach to the evaluation of insomnia. Future local studies examining issues related to insomnia management at the primary care level, as well as the broader perspective of insomnia evaluation at sleep medicine clinics, may help identify gaps in services and education needs.

Currently, diagnostic validity requires the use of attended PSG for primary sleep disorders and at least a type II portable PSG for SDB in patients with high pre-test probability of disease. ${ }^{(32)}$ While physicians may consider using commercially available and cheaper sleep monitoring tools (e.g. actigraphy devices and portable PSG), these are currently only acceptable as screening tools. Nonetheless, these may be viable primary care tools when used in conjunction with good sleep history-taking and examination, and a healthy index of suspicion.

\section{REFERENCES}

1. Lugaresi E, Cirignotta F, Zucconi M, Mondini, et al. Good and poor sleepers: an epidemiological survey of the San Marino population. In: Guilleminault C, Lugaresi E, editors. Sleep/wake Disorders: Natural history, epidemiology, and long term evolution. New York: Raven Press; 1983

2. Ganguli M, Reynolds CF, Gilby JE. Prevalence and persistence of sleep complaints in rural, older community sample: the MOVIES project. J Am Geriatr Soc 1996; 44:778-84.

3. Gislason T, Almqvist M. Somatic diseases and sleep complaints. An epidemiological study of 3,201 Swedish men. Acta Med Scand 1987; 221:475-81.

4. Maggi S, Langlois JA, Minicuci N, et al. Sleep complaints in community dwelling older persons: prevalence, associated factors, and reported causes. J Am Geriatr Soc 1998; 46:161-8.

5. Leger D, Poursain B, Neubauer D, Uchiyama M. An international survey of sleeping problems in the general population. Curr Med Res Opin 2008; 24:307-17.

6. Sanofi Synthelabo Singapore Pte Ltd. Survey on prevalence of insomnia and insomniac's coping behaviour in Singapore. The Straits Times 2002 Mar 30.

7. Yeo B, Perera IS, Kok LP, Tsoi WF. Insomnia in the community. Singapore Med J 1996; 37:282-4

8. Mellinger GD, Balter MB, Ulenhuth $\mathrm{EH}$. Insomnia and its treatment. Prevalence and correlates. Arch Gen Psychiatry 1985; 42:225-32.

9. Ford DE, Kamerow DB. Epidemiologic study of sleep disturbances and psychiatric disorders. An opportunity for prevention? JAMA 1989; 262:1479-84.

10. Breslau N, Roth T, Rosenthal L, Andreski P. Sleep disturbance and psychiatric disorders: a longitudinal epidemiologic study of young adults. Biol Psychiatry 1996; 39:411-8.

11. American Academy of Sleep Medicine. International Classification of Sleep Disorders: Diagnostic and Coding Manual. 2nd Edition. Westchester: American Academy of Sleep Medicine, 2005.

12. Lurie A. Obstructive sleep apnea in adults: epidemiology, clinical presentation, and treatment options. Adv Cardiol 2011; 46:1-42.

13. Luyster F, Buysse DJ, Strollo PJ Jr. Comorbid insomnia and obstructive sleep apnea: challenges for clinical practice and research. J Clin Sleep Med 2010; 6:196-204.

14. Krakow B, Melendrez D, Ferreira E, et al. Prevalence of insomnia symptoms in patients with sleep-disordered breathing. Chest 2001; 120:1923-9.

15. Chung KF. Insomnia subtypes and their relationship to daytime sleepiness in patients with obstructive sleep apnoea. Respiration 2005; 72:460-5.

16. Hui DS. Craniofacial profile assessment in patients with obstructive sleep apnoea. Sleep 2009; 32:11-2.

17. Hudson JL, Pope HG, Sullivan LE, et al. Good sleep, bad sleep: a meta-analysis of polysomnographic measures in insomnia, depression and narcolepsy. Biol Psychiatry 1992; 32:958-75.

18. Littner M, Hirshkowitz M, Kramer M, et al; Standards of Practice Committee of the American Academy of Sleep Medicine. Practice parameters for using polysomnography to evaluate insomnia: an update. Sleep 2003; 26:754-60.

19. Mahendran R, Subramaniam M, Chan YH. Psychiatric morbidity in patients referred to an insomnia clinic. Singapore Med J 2007; 48:163-5.

20. Rechtschaffen A, Kales A, eds. A manual of standardised terminology, techniques, and scoring system for sleep stages of human subjects. 
Bethesda: US Department of Health, Education, and Welfare Public Health Service, 1968.

21. Department of Statistics Singapore. Monthly Digest of Statistics Singapore [Online]. Available at: http://www.singstat.gov.sg. Accessed September 10, 2014.

22. Ministry of Health, Singapore. Prescribing of Benzodiazepines [Online]. Available at: https://www.moh.gov.sg/content/moh_web/ healthprofessionalsportal/doctors/guidelines/cpg_medical/2008/cpgmed_ prescribing_benzodiazepines.html. Accessed May 24, 2015.

23. Roth T. Insomnia: definition, prevalence, etiology, and consequences. J Clin Sleep Med 2007; 3(5 Suppl):S7-10.

24. Mahendran R. Characteristics of patients referred to an insomnia clinic. Singapore Med J 2001; 42:64-7.

25. Johns MW. A new method for measuring daytime sleepiness: the Epworth sleepiness scale. Sleep 1991; 14:540-5.

26. Glidewell RN, Roby EK, Orr WC. Is insomnia an independent predictor of obstructive sleep apnoea? J Am Board Fam Med 2012; 25:104-10.
27. Bjornsdottir E, Janson C, Gíslason T, et al. Insomnia in untreated sleep apnoea patients compared to controls. J Sleep Res 2012; 21:131-8.

28. Ohayon MM, Roth T. Prevalence of restless legs syndrome and periodic limb movement disorder in the general population. J Psychosom Res 2002; 5:547-54.

29. Schroder C, O'Hara R. Depression and obstructive sleep apnoea. Ann Gen Psychiatry 2005; 4:13.

30. Mahendran R, Subramaniam M, Chan YH. Medical students' behaviour, attitudes and knowledge of sleep medicine. Singapore Med J 2004; 45:587-9.

31. TOP. Assessment to Diagnosis: Insomnia. Toward Optimized Practice (TOP) Program [Online]. Available at: http://www.topalbertadoctors.org/ cpgs/?sid=18\&cpg.cats=79. Accessed May 24, 2015.

32. Collop NA, Anderson WM, Boehlecke B, et al. Clinical guidelines for the use of unattended portable monitors in the diagnosis of obstructive sleep apnea in adult patients. Portable Monitoring Task Force of the American Academy of Sleep Medicine. J Clin Sleep Med 2007; 7:737-47. 